\title{
Effect of Tumor Location on the Risk of Bilateral Central Lymph Node Metastasis in Unilateral I-4 cm Papillary Thyroid Carcinoma
}

Nan Liu

Bo Chen (iD)

Luchuan Li

Qingdong Zeng

Lei Sheng (D)

Bin Zhang

Bin Lv ID

Department of Thyroid Surgery, General Surgery, Qilu Hospital of Shandong University, Jinan, 2500 I2, Shandong, People's Republic of China
Correspondence: Bin Lv

Department of Thyroid Surgery, General Surgery, Qilu Hospital of Shandong

University, Jinan, 2500 I2, Shandong,

People's Republic of China

Email Ivbin@sdu.edu.cn
Purpose: Papillary thyroid carcinoma (PTC) has a high incidence of lymph node metastasis (LNM). Our aim was to determine whether tumor location is a useful feature to predict bilateral central lymph node metastasis (CLNM) in unilateral 1-4 cm PTC.

Patients and Methods: Data on unilateral 1-4 cm PTC patients from 2016 to 2019 were collected retrospectively. The clinical and pathological characteristics of the tumors and lymph nodes were analyzed statistically.

Results: The mean patient age was $49.1 \pm 12.3$ (23-73) years, and the majority were women $(\mathrm{n}=1334,75.4 \%)$. A total of 1767 patients were analyzed, and 256 (14.5\%) had bilateral CLNM. Tumor location was an independent risk factor in predicting bilateral CLNM $(p<0.001)$. The odds of bilateral CLNM were the highest in the near isthmus (OR 6.452, 95\% CI: $3.658-11.379, \mathrm{p}<0.001)$. In a multivariate regression model adjusting for other risk factors, near-isthmus tumors had the highest risk of bilateral CLNM (OR 7.319, 95\% CI: 3.844-13.933, $\mathrm{p}<0.001$ ), followed by lower lobe tumors (OR 2.338, 95\% CI: 1.315-4.155, $\mathrm{p}=0.004)$ and middle lobe tumors (OR 1.845, 95\% CI: 1.035-3.291, $\mathrm{p}=0.038$ ), compared to upper lobe tumors.

Conclusion: Tumor location is an independent risk factor in predicting the risk of bilateral CLNM. Near-isthmus tumors carry the highest risk of bilateral CLNM.

Keywords: papillary thyroid carcinoma, location, predictive factor, lymph node metastasis

\section{Introduction}

Papillary thyroid carcinoma (PTC) is the most common pathological type of thyroid cancer, and the incidence of PTC has dramatically increased in recent decades. ${ }^{1-3}$ The central neck compartment is the most common site of lymph node metastasis (LNM), and PTC patients have a high incidence. ${ }^{4-6}$ The central neck compartment includes the bilateral paratracheal regions, the prelaryngeal region and the pretracheal region. A total of $40-60 \%$ of PTCs have central lymph node metastasis (CLNM), and bilateral CLNM was even found in $8.1-36.6 \%$ of unilateral PTCs. ${ }^{7-14}$ Previous studies have found that LNM can influence local recurrence and distant metastasis. ${ }^{15-18}$ However, central compartment neck dissection (CCND) can increase the risk of postoperative hypocalcemia. In terms of unilateral PTC, bilateral CCND should only be performed in patients with a high risk of bilateral CLNM.

To date, risk factors for bilateral CLNM include male sex, $>1 \mathrm{~cm}$ PTC, extrathyroidal extension, lateral neck LNM, and ipsilateral CLNM. ${ }^{8,11-14,19,20}$ However, little attention has been given to the effect of tumor location on the risk of bilateral 
CLNM. Therefore, we conducted this study to test the risk of bilateral CLNM based on tumor location in unilateral PTC in order to provide valuable screening criteria for thyroid surgeons.

\section{Patients and Methods}

\section{Patient Selection}

This retrospective study collected data on patients proven to have unilateral PTC by preoperative highresolution ultrasound and fine needle aspiration at Qilu Hospital of Shandong University between January 2016 and March 2019. Only patients with 1$4 \mathrm{~cm}$ PTC were selected because $<1 \mathrm{~cm}$ PTC has a low risk of bilateral CLNM, and it is difficult to pinpoint the location of $>4 \mathrm{~cm}$ PTC. All patients underwent total thyroidectomy and bilateral CCND with/without lateral neck dissection. All patients underwent highresolution ultrasound and fine needle aspiration before surgery. This study was approved by the Medical Ethics Committee of Qilu Hospital of Shandong
University (Project identification code: 2018149). All patients were communicated with written and oral information and patient consents to review their medical records were acquired. Patient data are not publicly available due to the sensitive nature of the information but are available from the corresponding author on reasonable request. This study was registered in the Research Registry (UIN: researchregistry5292). The study design is shown in the flowchart (Figure 1).

The following exclusion criteria were adopted: history of thyroidectomy, bilateral PTC proven by postoperative histology, tumor located within the isthmus or pyramidal lobe, and distant metastasis. Multifocal unilateral PTC was allowed. The patients were divided into two groups based on the status of bilateral CLNM. This study complies with the Declaration of Helsinki.

\section{Surgical Procedure}

All surgeries were performed by the same two experienced thyroid surgeons. The standardized CCND was as follows:

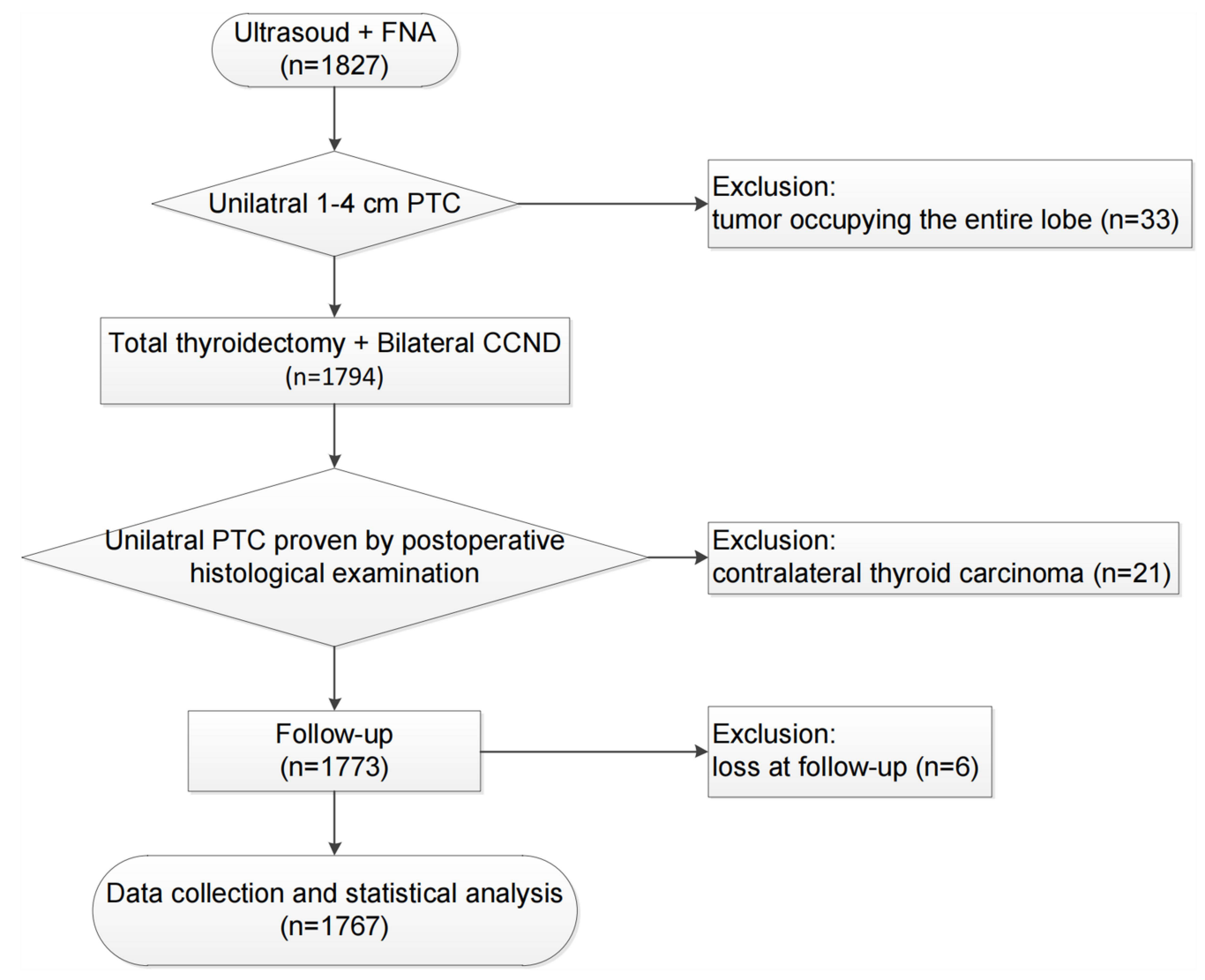

Figure I The flowchart of the study.

Abbreviations: FNA, fine needle aspiration; PTC, papillary thyroid carcinoma; and CCND, central compartment neck dissection. 
the demarcation of the prelaryngeal region was that the superior border of the thyroid cartilage served as the superior bound and the inferior border of the cricoid cartilage as the inferior bound; the demarcation of the pretracheal region was that the inferior border of the isthmus served as the superior bound, the innominate artery served as the inferior bound and the tangent of the lateral wall of the trachea served as the lateral bound; and the demarcation of the paratracheal region was that the common carotid artery served as the lateral bound and the lateral wall of the trachea served as the medial bound. Specimens from different regions were sent for histological examination.

\section{Clinical Assessment}

The following indicators were collected and analyzed: age, gender, place of residence, education level, tumor location, tumor size, multifocality, aggressive pathology, intraglandular dissemination, extrathyroidal extension, number of metastatic lymph nodes, lateral neck LNM, underlying conditions of Hashimoto's thyroiditis, and $B R A F \mathrm{~V} 600 \mathrm{E}$ mutation. The primary tumor location was subjectively categorized as the upper lobe, middle lobe, lower lobe and near isthmus (Figure 2A). A near-isthmus tumor was defined as a tumor with the border exceeding the lateral wall of the trachea (Figure 2B). Tumors occupying both the middle and the lower thyroid lobes were included in the lower thyroid tumor category. Tumors occupying the entire thyroid lobe were excluded from the analysis. Intraglandular dissemination meant that cancerous embolisms with heterotypic cells and psammoma bodies were found in the lymphatic vessels surrounding a major carcinoma. ${ }^{21}$ Tall cell variant, solid variant, columnar cell variant and hobnail variant were included in aggressive pathology.

\section{Statistical Analysis}

Assuming a 10\% loss due to follow-up, at least 496 patients were needed to achieve $90 \%$ power at a $5 \%$ significance level on the basis of our practical experience and the rate of bilateral CLNM described in previous publications. $^{10-14}$

Statistical analyses were performed using IBM SPSS Statistics for Windows, version 21.0 (IBM Corp., Armonk, N.Y., USA). We used descriptive statistics to summarize the demographics and clinicopathological characteristics. Continuous variables are presented as the mean \pm standard deviation (SD) and were compared using the $t$-test or Mann-Whitney $U$-test. Conversely, categorical variables are presented as frequencies (percentages) and were compared using the chi-squared test or Fisher's exact test. Before multivariate logistic regression analysis, a collinearity test was conducted for variables that may influence the risk of bilateral CLNM in univariate analysis. In the multivariate analysis, binomial logistic regression analysis was performed to estimate the odds ratio (OR) and the $95 \%$ confidence interval $(95 \% \mathrm{CI})$ of bilateral CLNM.
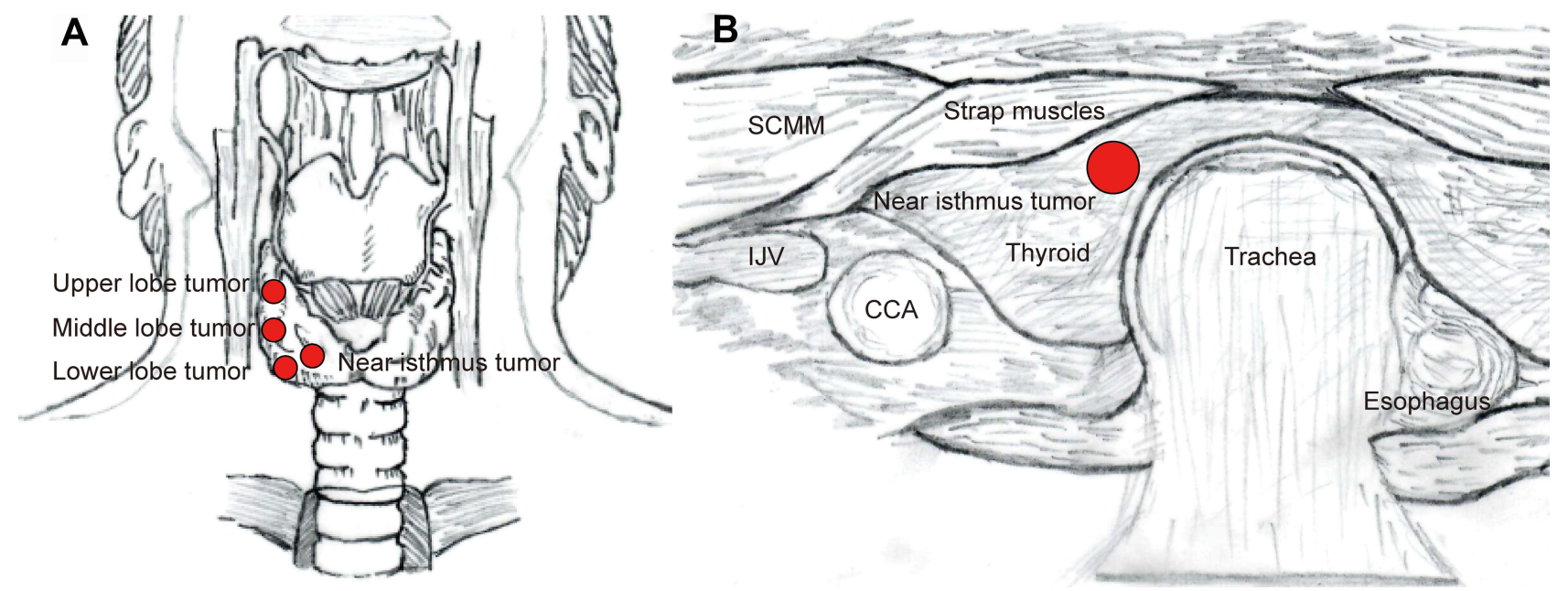

Figure 2 Schematic representation of different localization methods of PTC tumors. A. Longitudinal coronal location. B. Transversal sagittal location. Abbreviations: IJV, internal jugular vein; CCA, common carotid artery; and SCMM sternocleidomastoid muscle. 
Table I The Demographic and Clinicopathological Characteristics of the Patients

\begin{tabular}{|c|c|c|}
\hline Characteristic & Patients $n=1767$ & (\%) \\
\hline Age (min-max) & $49.1 \pm 12.3(23-73)$ & \\
\hline \multicolumn{3}{|l|}{ Sex } \\
\hline Male & 433 & 24.5 \\
\hline Female & 1334 & 75.4 \\
\hline \multicolumn{3}{|l|}{ Place of residence } \\
\hline City & 1123 & 63.6 \\
\hline Village & 644 & 36.4 \\
\hline \multicolumn{3}{|l|}{ Education level } \\
\hline Primary school or high school & 516 & 29.2 \\
\hline College or university & $|25|$ & 70.8 \\
\hline Tumor size $(\mathrm{mm})$ & $19.1 \pm 7.4$ & \\
\hline Aggressive pathology & 97 & 5.4 \\
\hline Multifocality & 256 & 14.5 \\
\hline Intraglandular dissemination & 47 & 2.7 \\
\hline Extrathyroidal extension & 931 & 52.7 \\
\hline \multicolumn{3}{|l|}{ Tumor laterality } \\
\hline Right lobe & 1123 & 63.6 \\
\hline Left lobe & 644 & 36.4 \\
\hline \multicolumn{3}{|l|}{ Tumor location } \\
\hline Upper lobe & 190 & 10.7 \\
\hline Middle lobe & 793 & 44.8 \\
\hline Lower lobe & 638 & 36.1 \\
\hline Near isthmus & 146 & 8.2 \\
\hline \multicolumn{3}{|l|}{ CLNM (pNIa) } \\
\hline Ipsilateral paratracheal LNM & 851 & 48.2 \\
\hline Bilateral CLNM & 256 & 14.5 \\
\hline Pretracheal LNM & 92 & 5.2 \\
\hline Prelaryngeal LNM & 28 & 1.6 \\
\hline Lateral neck LNM, n (\%) & 207 & 11.7 \\
\hline Hashimoto's thyroiditis & 384 & 21.7 \\
\hline BRAF mutation $(+)$ & 1306 & 73.9 \\
\hline
\end{tabular}

Abbreviations: CLNM, central lymph node metastasis; LNM, lymph node metastasis.

To further determine the roles of the risk factors for different age patients, we performed a subgroup analysis using the cutoff of 55 years of age of the eighth edition of the AJCC/TNM cancer staging system. ${ }^{22}$ One-way analysis of variance (ANOVA) was used to compare the effects of different tumor locations on tumor size and the number of metastatic lymph nodes.
A $p$ value $<0.05$ was considered statistically significant, and the reported $\mathrm{p}$ values were two-sided.

\section{Results}

Of the 1827 initial PTC patients, 33 patients with tumors occupying the entire lobe were excluded, and 6 patients were lost to follow-up. Twenty-one patients were excluded due to bilateral PTC verified by postoperative histology. A total of 1767 patients were ultimately analyzed (Figure 1). The demographic, clinical and pathological characteristics of all patients are shown in Table 1. The mean patient age was 49.1 \pm 12.3 (range 23-73) years. The majority of patients were women $(\mathrm{n}=1334,75.4 \%)$. The tumor size ranged from 11 to $40 \mathrm{~mm}$ (average $19.1 \pm 7.4 \mathrm{~mm}$ ). The tumors were mostly distributed in the right lobes $(n=1123$, $63.6 \%)$ and less frequently in the left lobes $(n=644$, $36.4 \%$ ). These tumors were located in the upper lobe in $190(10.7 \%)$ patients, middle lobe in $793(44.8 \%)$ patients, lower lobe in 638 (36.1\%) patients, and near isthmus in $146(8.2 \%)$ patients. There was ipsilateral paratracheal LNM in $851(48.2 \%)$ patients and bilateral CLNM in 256 (14.5\%) patients. Additionally, pretracheal and prelaryngeal LNM were found in 92 and 28 patients, respectively, and lateral neck LNM was found in $207(11.7 \%)$ patients.

In the univariate analysis (Table 2), patients with bilateral CLNM had more ipsilateral paratracheal $(\mathrm{p}<0.001)$ and pretracheal $(\mathrm{p}=0.001)$ metastatic lymph nodes. In addition, being male $(\mathrm{p}<0.001)$, tumor size $(\mathrm{p}=0.001)$, aggressive pathology $(\mathrm{p}<0.001)$ and lateral neck LNM $(p=0.002)$ were more likely to be associated with bilateral CLNM. Tumor location was significantly different between the positive and negative bilateral CLNM groups $(\mathrm{p}<0.001)$. Positive bilateral CLNM accounted for $10.5 \%(20 / 190)$ of the upper lobe tumors, $11.0 \%(87 / 793)$ of the middle lobe tumors, $13.5 \%(86 / 638)$ of the lower lobe tumors and $43.2 \%$ $(63 / 146)$ of the near-isthmus tumors (Table 2).

The number of metastatic lymph nodes was removed from multivariate logistic regression analysis because of collinearity with other risk factors. In the final multivariate analysis, male sex (OR 3.833, 95\% CI: 2.832-5.186, $\mathrm{p}<0.001$ ), tumor size (OR 1.392, 95\% CI: $1.011-1.916$, $\mathrm{p}=0.043$ ), aggressive pathology (OR 9.159, 95\% CI: 5.717-14.673, $\mathrm{p}<0.001)$ and lateral neck LNM (OR 
Table 2 Clinical and Pathological Characteristics Related to Bilateral CLNM in Univariate Analysis

\begin{tabular}{|c|c|c|c|}
\hline Characteristics & $\begin{array}{l}\begin{array}{c}\text { Positive Bilateral CLNM } \\
n=256\end{array}\end{array}$ & $\begin{array}{l}\text { Negative Bilateral CLNM } \\
\qquad n=|5| \mid\end{array}$ & p value \\
\hline Age (min-max) & $50.1 \pm 11.3(23-69)$ & $48.9 \pm 12.5(27-73)$ & 0.135 \\
\hline Gender & & & $<0.001 *$ \\
\hline Male, n (\%) & $|3|(29.6 \%)$ & $302(70.4 \%)$ & \\
\hline Female, n (\%) & 125 (9.4\%) & 1209 (90.6\%) & \\
\hline Place of residence & & & 0.100 \\
\hline City, n (\%) & I5I (I3.4\%) & $972(86.6 \%)$ & \\
\hline Village, n (\%) & $105(16.3 \%)$ & $539(83.7 \%)$ & \\
\hline Education level & & & 0.169 \\
\hline Primary or high school & $84(16.3 \%)$ & $432(83.7 \%)$ & \\
\hline College or university & $172(13.7 \%)$ & $1079(86.3 \%)$ & \\
\hline Tumor size $(\mathrm{mm})$ & $20.9 \pm 7.9$ & $18.8 \pm 7.2$ & $0.001 *$ \\
\hline Aggressive pathology, n (\%) & $56(57.7 \%)$ & 41 (42.3\%) & $<0.001 *$ \\
\hline Multifocality, n (\%) & $43(16.8 \%)$ & $213(83.2 \%)$ & 0.256 \\
\hline Intraglandular dissemination, $\mathrm{n}(\%)$ & $5(10.6 \%)$ & $42(89.4 \%)$ & 0.447 \\
\hline Extrathyroidal extension, n (\%) & $129(13.9 \%)$ & $802(86.1 \%)$ & 0.426 \\
\hline \multicolumn{4}{|l|}{ Tumor laterality } \\
\hline Right lobe & 152 (13.5\%) & 971 (86.5\%) & 0.133 \\
\hline Left lobe & $104(16.1 \%)$ & $540(83.9 \%)$ & \\
\hline Tumor location & & & $<0.00 I^{*}$ \\
\hline Upper lobe & $20(10.5 \%)$ & $170(89.5 \%)$ & \\
\hline Middle lobe & 87 (11.0\%) & 706 (89.0\%) & \\
\hline Lower lobe & $86(13.5 \%)$ & $552(86.5 \%)$ & \\
\hline Near isthmus & $63(43.2 \%)$ & $83(56.8 \%)$ & \\
\hline $\begin{array}{l}\text { Number of metastatic lymph nodes in ipsilateral paratracheal } \\
\text { region }\end{array}$ & $6.9 \pm 1.5$ & $2.0 \pm 2.7$ & $<0.001 *$ \\
\hline Number of metastatic lymph nodes in pretracheal region & $0.3 \pm 0.9$ & $0.1 \pm 0.6$ & $0.001 *$ \\
\hline Number of metastatic lymph nodes in prelaryngeal region & $0.1 \pm 0.4$ & $0.03 \pm 0.3$ & 0.114 \\
\hline Total number of metastatic lymph nodes & $7.2 \pm 2.3$ & $2.2 \pm 3.0$ & $<0.001 *$ \\
\hline Lateral neck LNM, n (\%) & $45(21.7 \%)$ & $162(78.3 \%)$ & $0.002^{*}$ \\
\hline Hashimoto's thyroiditis, $\mathrm{n}(\%)$ & $48(12.5 \%)$ & $336(87.5 \%)$ & 0.211 \\
\hline BRAF mutation+, $\mathrm{n}(\%)$ & $179(13.7 \%)$ & 1127 (86.3\%) & 0.116 \\
\hline
\end{tabular}

Note: $* p<0.05$.

Abbreviations: CLNM, central lymph node metastasis; LNM, lymph node metastasis.

2.266, 95\% CI: $1.482-3.464, \mathrm{p}<0.001)$ were independent predictive factors of bilateral CLNM (Table 3). Tumor location was also an independent predictive factor of bilateral CLNM after adjusting for the above risk factors.
Compared to upper lobe tumors, near-isthmus tumors continued to demonstrate the highest risk of bilateral CLNM in a multivariate regression model (OR 7.319, 95\% CI: 3.844-13.933, $\mathrm{p}<0.001$ ), followed by lower lobe tumors 
Table 3 Multivariate Logistic Regression Analysis for Bilateral CLNM in All Patients

\begin{tabular}{|l|c|c|c|}
\hline Variables & OR & $\mathbf{9 5 \%} \mathbf{~ C I}$ & $\mathbf{P}$ value \\
\hline $\begin{array}{l}\text { Tumor location } \\
\text { Upper lobe (reference) }\end{array}$ & - & - & \\
Middle lobe & 1.845 & $1.035-3.291$ & - \\
Lower lobe & 2.338 & $1.315-4.155$ & 0.038 \\
Near isthmus & 7.319 & $3.844-13.933$ & 0.004 \\
\hline Tumor size & 1.392 & $1.011-1.916$ & 0.043 \\
\hline Male & 3.833 & $2.832-5.186$ & $<0.001$ \\
\hline Aggressive pathology & 9.159 & $5.717-14.673$ & $<0.001$ \\
\hline Lateral neck LNM & 2.266 & $1.482-3.464$ & $<0.001$ \\
\hline
\end{tabular}

Abbreviations: CLNM, central lymph node metastasis; LNM, lymph node metastasis.

(OR 2.338, 95\% CI: 1.315-4.155, $\mathrm{p}=0.004)$ and middle lobe tumors (OR 1.845, 95\% CI: 1.035-3.291, $\mathrm{p}=0.038$ ) (Table 3).

As shown in the subgroup analysis, near-isthmus tumors still had the highest risk of bilateral CLNM among patients in the $<55$ years group (OR 5.508, 95\% CI: $2.371-12.793, \mathrm{p}<0.001)$ and $\geq 55$ years group (OR 10.811, 95\% CI: 3.895-30.008, p<0.001) (Table 4). Male sex and aggressive pathology remained independent predictive factors of bilateral CLNM in both subgroups. Tumor size was an independent predictive factor of bilateral CLNM in patients $<55$ years but not in patients $\geq 55$ years. However, lateral neck LNM was an independent predictive factor of bilateral CLNM in patients $\geq 55$ years but not in patients $<55$ years.

Compared with near-isthmus tumors, the tumors located in the lower lobe $(p=0.021)$ and middle lobe $(p=0.003)$ were larger, but the tumors located in the upper lobe were smaller $(p=0.008)$ (Figure 3). Additionally, near-isthmus tumors had more pretracheal metastatic lymph nodes than upper $(\mathrm{p}=0.001)$ and middle lobe tumors $(p=0.027)$ but not lower lobe tumors $(p=0.07)$ (Figure 4B). More ipsilateral paratracheal metastatic lymph nodes were found in lower lobe tumors than in upper lobe tumors $(\mathrm{p}=0.03)$ (Figure $4 \mathrm{~A})$. Both near isthmus and lower lobe tumors had more total metastatic lymph nodes than upper lobe tumors $(\mathrm{p}=0.027$ and 0.008 , respectively) (Figure 4D). However, the prelaryngeal metastatic lymph nodes of all tumor locations were not significantly different (Figure 4C).

\section{Discussion}

This study shows that tumor location is an independent risk factor for bilateral CLNM in unilateral $1-4 \mathrm{~cm}$ PTC. While near-isthmus tumors were the least frequent $(8.2 \%)$, they had the highest risk of bilateral CLNM. Furthermore, near-isthmus tumors were smaller and had more metastatic lymph nodes in the pretracheal region. Tumors located in the upper lobe were associated with the lowest risk of bilateral CLNM.

The risk factors for bilateral CLNM, such as male sex, tumor size, extrathyroidal extension, ipsilateral

Table 4 Multivariate Logistic Regression Analysis for Bilateral CLNM Grouping Patients by Age

\begin{tabular}{|c|c|c|c|c|c|c|}
\hline \multirow[t]{2}{*}{ Variables } & \multicolumn{3}{|c|}{ Age $<55$ Group } & \multicolumn{3}{|c|}{ Age $\geq 55$ Group } \\
\hline & OR & $95 \% \mathrm{Cl}$ & $\mathbf{p}$ & OR & $95 \% \mathrm{Cl}$ & $\mathbf{p}$ \\
\hline \multicolumn{7}{|l|}{ Tumor location } \\
\hline Upper (reference) & - & - & - & - & - & - \\
\hline Middle & 1.907 & $0.908-4.004$ & 0.088 & 1.691 & $0.657-4.353$ & 0.276 \\
\hline Lower & 1.881 & $0.888-3.987$ & 0.099 & 3.065 & I.223-7.682 & 0.017 \\
\hline Near isthmus & 5.508 & $2.37 I-12.793$ & $<0.001$ & 10.811 & $3.895-30.008$ & $<0.001$ \\
\hline Tumor size & 1.666 & $1.105-2.513$ & 0.015 & 1.081 & $0.640-1.824$ & 0.771 \\
\hline Male & 3.947 & $2.665-5.847$ & $<0.001$ & 3.867 & $2.385-6.269$ & $<0.001$ \\
\hline Aggressive pathology & 9.472 & $5.150-17.421$ & $<0.001$ & 8.146 & $3.790-17.504$ & $<0.001$ \\
\hline Lateral neck LNM & 1.738 & $0.987-3.060$ & 0.055 & 3.455 & I.770-6.745 & $<0.001$ \\
\hline
\end{tabular}

Abbreviations: CLNM, central lymph node metastasis; LNM, lymph node metastasis. 


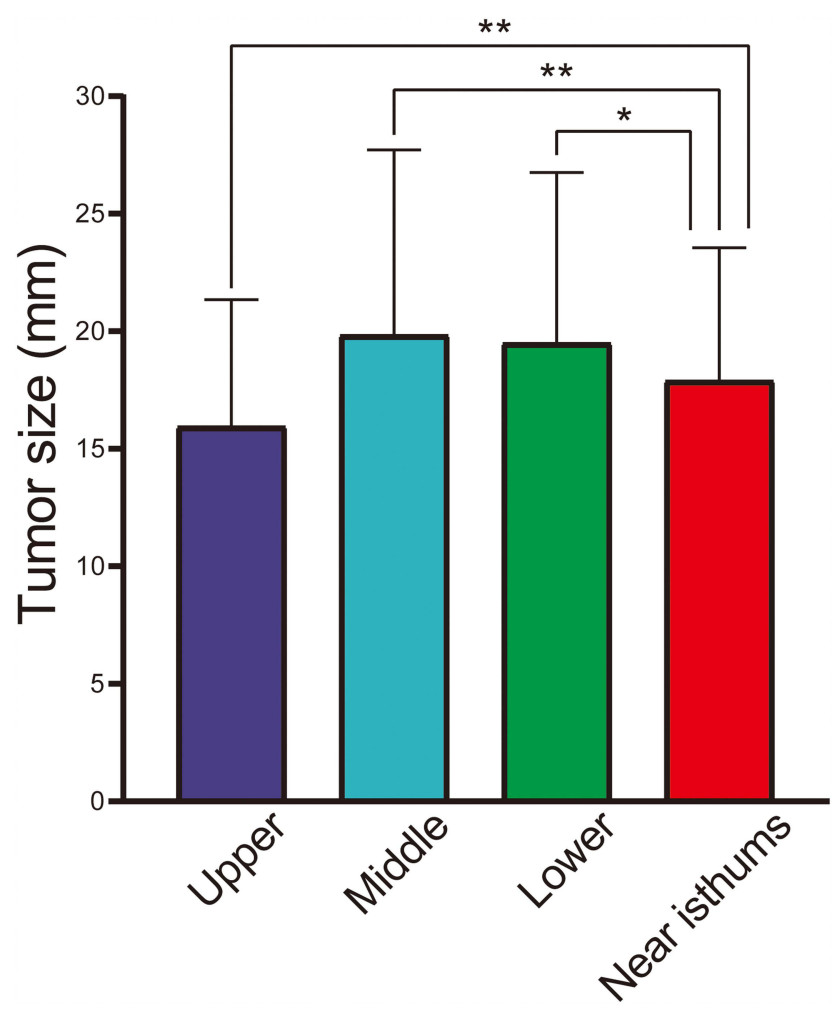

Figure 3 Tumor size in different tumor locations in ANOVA analyses. ${ }^{*} \mathrm{p}<0.05$, and ** $\mathrm{p}<0.01$.

CLNM, and lateral neck LNM, have been reported in some published literature. ${ }^{8,11-14,19,20}$ This study also confirmed that male sex, aggressive pathology, lateral neck LNM and tumor size were predictors of bilateral CLNM. However, extrathyroidal extension was not found to be an independent risk factor for bilateral CLNM. This is probably because most extrathyroidal extension was detected only by microscopy and had no impact on LNM. When tumor location was added to the above model with the adjustment of other known features, it was still an independent risk factor to predict bilateral CLNM. Some studies have reported that the risk of cervical LNM was associated with tumor location in PTC. ${ }^{23-26}$ However, there is no research regarding the effect of tumor location on the risk of bilateral CLNM. One study showed that isthmus tumors were more likely to have CLNM than tumors in other locations. ${ }^{26}$ Other studies demonstrated that the risk of lateral neck LNM was higher in upper pole tumors, and the risk of CLNM was much lower. ${ }^{23-25}$

It is not clear why near-isthmus tumors are at a higher risk of bilateral CLNM. The possible mechanisms could be that PTC located in the near isthmus has similar characteristics as those located in the isthmus. Despite the low incidence and small tumor size, PTC arising from the isthmus tends to be more aggressive and has a poorer prognosis. ${ }^{27-32}$ Additionally, there are some data suggesting that PTC located in the isthmus is more likely to have $\mathrm{LNM}^{33}{ }^{33}$ multifocality, ${ }^{27,28}$ capsular invasion and extrathyroidal extension. ${ }^{34}$ The more aggressive behavior of isthmic PTC is likely related to the thin shape of the isthmus, which may be more likely to invade the thyroid capsule and adjacent tissues. Additionally, the lymph from the isthmus more frequently spreads to the pretracheal or prelaryngeal region and then drains to the paratracheal region. ${ }^{35-37}$ In this study, more pretracheal metastatic lymph nodes were found in patients with near-isthmus tumors.

The aim of this study was to assess the effect of tumor location on the risk of bilateral CLNM. According to the results, near-isthmus tumors had the highest risk of bilateral CLNM and more metastatic lymph nodes in the pretracheal region. Therefore, the identification of tumor location and intraoperative frozen section examinations of lymph nodes in the pretracheal region may be useful in determining the appropriate extent of CCND. Some studies have reported that intraoperative frozen section examinations of ipsilateral lymph nodes have high sensitivity, specificity and accuracy in predicting contralateral nodal status. ${ }^{14,38-40}$

However, one limitation of this study was the retrospective nature, which induced the risk of selection bias. The other limitation was that $B R A F$ gene mutation is not the only genetic factor that may impact LNM. Further studies on the molecular mechanism of LNM are necessary. Additionally, long-term postoperative follow-up data are also needed.

\section{Conclusion}

In summary, our data indicate that tumor location may be an independent factor for the risk of bilateral CLNM in unilateral 1-4 cm PTC. Near-isthmus tumors have the highest risk of bilateral CLNM while tumors in the upper lobe have the lowest risk. Furthermore, thyroid tumors located in the near isthmus have more metastatic lymph nodes in the pretracheal region. These data may help to carefully evaluate nearisthmus tumors in thyroid surgery and determine the treatment plan for surveillance or the ultimate extent of CCND. 
A

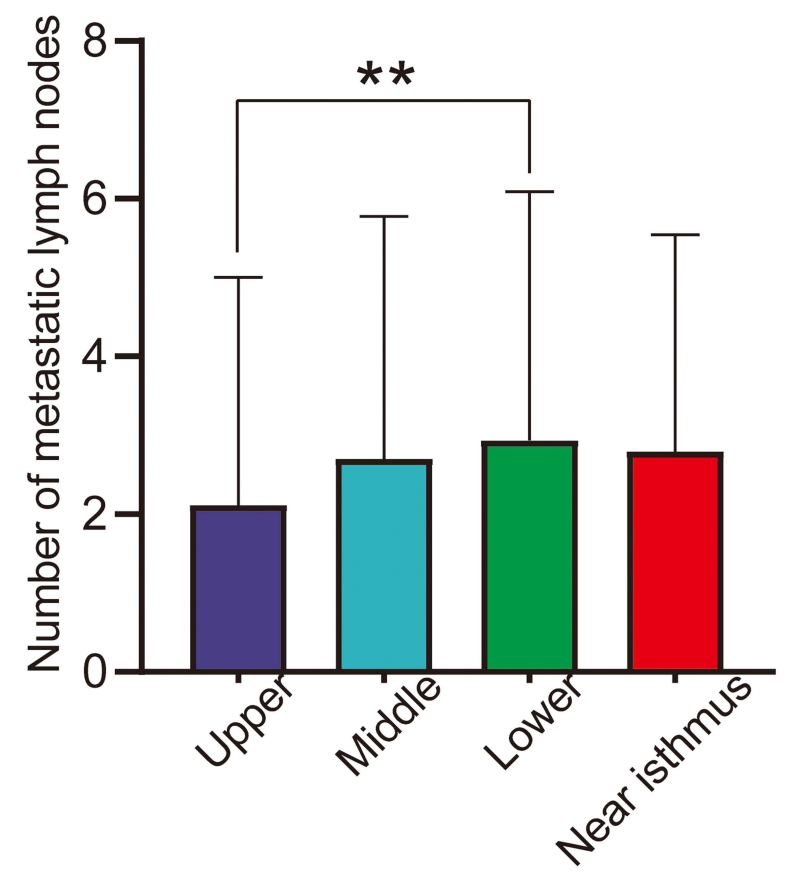

C

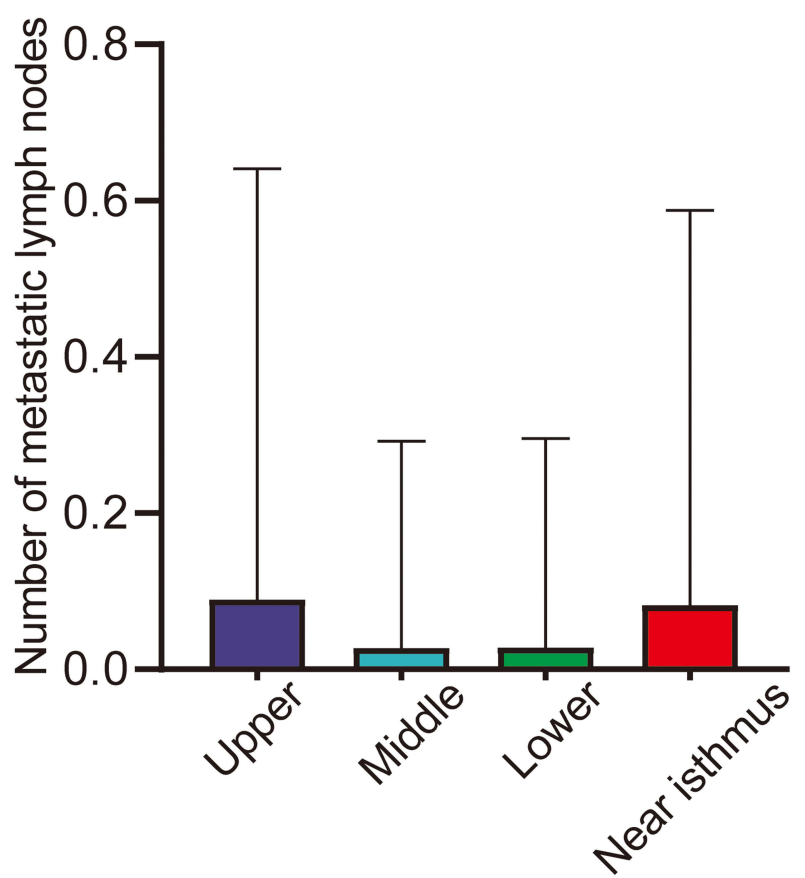

B

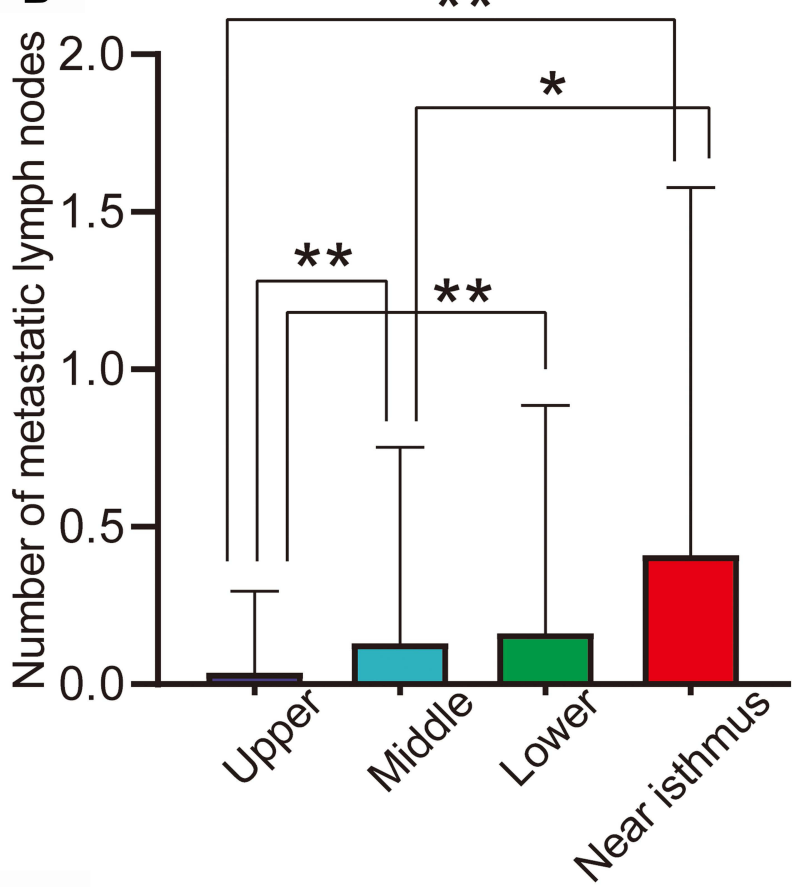

D

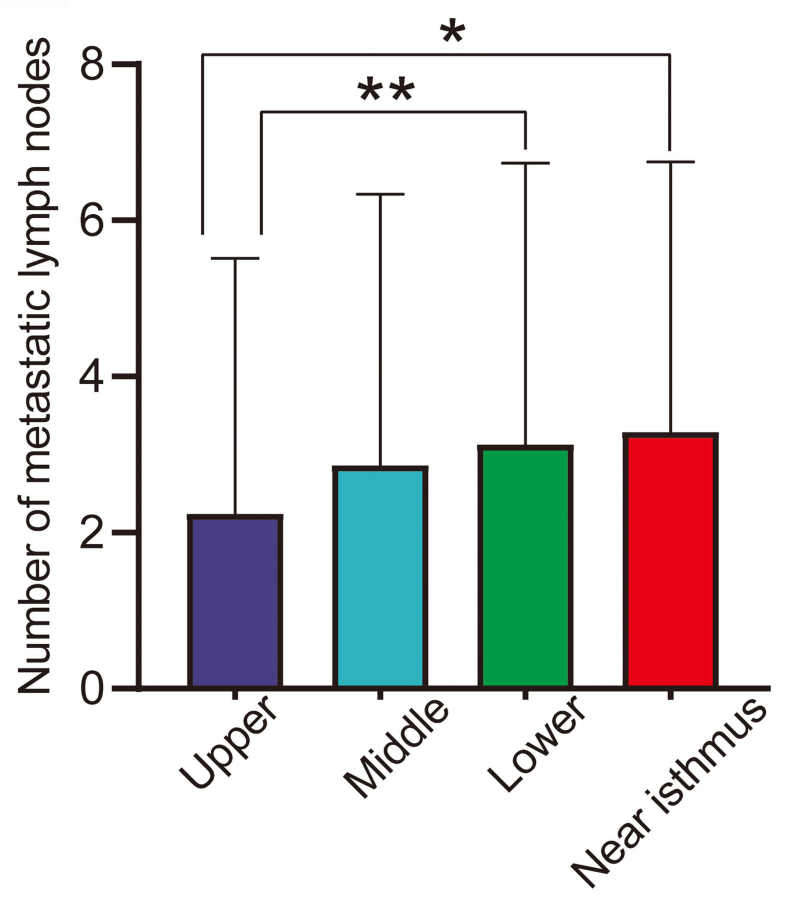

Figure 4 The number of metastatic lymph nodes in different tumor locations in ANOVA analyses. (A) Number of metastatic lymph nodes in the ipsilateral paratracheal region. (B) Number of metastatic lymph nodes in the pretracheal region. (C) Number of metastatic lymph nodes in the prelaryngeal region. (D) Total number of metastatic lymph nodes in three regions. $* \mathrm{p}<0.05$ and $* * \mathrm{p}<0.01$.

\section{Abbreviations}

PTC, papillary thyroid carcinoma; LNM, lymph node metastasis; CLNM, central lymph node metastasis; CCND, central compartment neck dissection.

\section{Acknowledgments}

This research was supported by the Key Technology Research and Development Program of Shandong Province (grant No. 2019GSF108072). 


\section{Disclosure}

The authors have no conflicts of interest to declare that they are relevant to the content of this article.

\section{References}

1. Udelsman R, Zhang Y. The epidemic of thyroid cancer in the United States: the role of endocrinologists and ultrasounds. Thyroid. 2014;24 (3):472-479. doi:10.1089/thy.2013.0257

2. La Vecchia C, Malvezzi M, Bosetti C, et al. Thyroid cancer mortality and incidence: a global overview. Int $J$ Cancer. 2015;136 (9):2187-2195. doi:10.1002/ijc.29251

3. Haugen BR, Alexander EK, Bible KC, et al. 2015 American Thyroid Association Management guidelines for adult patients with thyroid nodules and differentiated thyroid cancer: the american thyroid association guidelines task force on thyroid nodules and differentiated thyroid cancer. Thyroid. 2016;26(1):1-133. doi:10.1089/ thy. 2015.0020

4. Kouvaraki MA, Shapiro SE, Fornage BD, et al. Role of preoperative ultrasonography in the surgical management of patients with thyroid cancer. Surgery. 2003;134(6):946-954; discussion 954-955. doi:10.1016/s0039-6060(03)00424-0

5. Hughes DT, White ML, Miller BS, Gauger PG, Burney RE, Doherty GM. Influence of prophylactic central lymph node dissection on postoperative thyroglobulin levels and radioiodine treatment in papillary thyroid cancer. Surgery. 2010;148(6):1100-1106; discussion 1006-1007. doi:10.1016/j.surg.2010.09.019

6. Randolph GW, Duh Q-Y, Heller KS, et al. The prognostic significance of nodal metastases from papillary thyroid carcinoma can be stratified based on the size and number of metastatic lymph nodes, as well as the presence of extranodal extension. Thyroid. 2012;22 (11):1144-1152. doi:10.1089/thy.2012.0043

7. Lee KE, Chung IY, Kang E, et al. Ipsilateral and contralateral central lymph node metastasis in papillary thyroid cancer: patterns and predictive factors of nodal metastasis. Head Neck. 2013;35(5):672-676. doi: 10.1002/hed.23016

8. Eun YG, Lee YC, Kwon KH. Predictive factors of contralateral paratracheal lymph node metastasis in papillary thyroid cancer: prospective multicenter study. Otolaryngol Head Neck Surg. 2014;150 (2):210-215. doi:10.1177/0194599813514726

9. Delogu D, Pisano IP, Pala C, et al. Prophylactic central neck lymphadenectomy in high risk patients with $\mathrm{T} 1$ or $\mathrm{T} 2$ papillary thyroid carcinoma: is it useful? Ann Ital Chir. 2014;85(3):225-229.

10. Zhou B, Qin J. High-risk factors for lymph node metastasis in contralateral central compartment in unilateral papillary thyroid carcinoma (cT1N0). Eur J Surg Oncol. 2021;47(7):882-887. doi:10.1016/j.ejso.2020.10.018

11. Hei H, Song Y, Qin J. A nomogram predicting contralateral central neck lymph node metastasis for papillary thyroid carcinoma. $J$ Surg Oncol. 2016;114(6):703-707. doi:10.1002/jso.24403

12. Wei T, Chen R, Zou X, Liu F, Li Z, Zhu J. Predictive factors of contralateral paratracheal lymph node metastasis in unilateral papillary thyroid carcinoma. Eur J Surg Oncol. 2015;41(6):746-750. doi:10.1016/j.ejso.2015.02.013

13. Chen Q, Wei T, Wang X-L, Li Z-H, Du Z-H, Zhu J-Q. The total number of prelaryngeal and pretracheal lymph node metastases: is it a reliable predictor of contralateral central lymph node metastasis in papillary thyroid carcinoma? J Surg Res. 2017;214:162-167. doi:10.1016/j.jss.2015.02.056

14. Zhou L, Li H, Liang W, Gao C, Chen B. Pretracheal-laryngeal lymph nodes in frozen section predicting contralateral paratracheal lymph nodes metastasis. Eur J Surg Oncol. 2020;46(10 Pt A):1829-1834. doi:10.1016/j.ejso.2020.06.048
15. Podnos YD, Smith D, Wagman LD, Ellenhorn JDI. The implication of lymph node metastasis on survival in patients with well-differentiated thyroid cancer. Am Surg. 2005;71(9):731-734. doi:10.1177/000313480507100907

16. Leboulleux S, Rubino C, Baudin E, et al. Prognostic factors for persistent or recurrent disease of papillary thyroid carcinoma with neck lymph node metastases and/or tumor extension beyond the thyroid capsule at initial diagnosis. $J$ Clin Endocrinol Metab. 2005;90(10):5723-5729. doi:10.1210/jc.2005-0285

17. Sugitani I, Kasai N, Fujimoto Y, Yanagisawa A. A novel classification system for patients with PTC: addition of the new variables of large $(3 \mathrm{~cm}$ or greater) nodal metastases and reclassification during the follow-up period. Surgery. 2004;135(2):139-148. doi:10.1016/ s0039-6060(03)00384-2

18. Adam MA, Pura J, Goffredo P, et al. Presence and number of lymph node metastases are associated with compromised survival for patients younger than age 45 years with papillary thyroid cancer. J Clin Oncol. 2015;33(21):2370-2375. doi:10.1200/ JCO.2014.59.8391

19. Roh J-L, Kim J-M, Park CI. Central lymph node metastasis of unilateral papillary thyroid carcinoma: patterns and factors predictive of nodal metastasis, morbidity, and recurrence. Ann Surg Oncol. 2011;18(8):2245-2250. doi:10.1245/s10434-011-1600-z

20. Miao S, Mao X, Pei R, et al. Predictive factors for different subgroups of central lymph node metastasis in unilateral papillary thyroid carcinoma. ORL J Otorhinolaryngol Relat Spec. 2013;75 (5):265-273. doi:10.1159/000354267

21. Jin H, Yan H, Tang H, Zheng M, Wu C, Liu J Internal spreading of papillary thyroid carcinoma: a case report and systemic Review. Case Rep Endocrinol. 2018;2018:7618456. doi:10.1155/2018/7618456.

22. Tuttle M, Morris LF, Haugen B, et al. Thyroid-differentiated and anaplastic carcinoma. In: Amin MB, Edge SB, Greene F, et al., editors. AJCC Cancer Staging Manual. 8th ed. New York: Springer International Publishing; 2017:873-891.

23. Sancaktar ME, Saylam G, Öcal B, et al. Possible prediction of patterns of cervical lymph node spread based on primary tumor location in papillary thyroid carcinomas. Turk J Med Sci. 2019;49 (1):217-221. doi:10.3906/sag-1807-79

24. Back K, Kim JS, Kim J-H, Choe J-H. Superior located papillary thyroid microcarcinoma is a risk factor for lateral lymph node metastasis. Ann Surg Oncol. 2019;26(12):3992-4001. doi:10.1245/ s10434-019-07587-2

25. Lee YS, Shin S-C, Lim Y-S, et al. Tumor location-dependent skip lateral cervical lymph node metastasis in papillary thyroid cancer. Head Neck. 2014;36(6):887-891. doi:10.1002/hed.23391

26. Zhang -T-T, Qi X-Z, Chen J-P, et al. The association between tumor's location and cervical lymph nodes metastasis in papillary thyroid cancer. Gland Surg. 2019;8(5):557-568. doi:10.21037/gs.2019.10.02

27. Goldfarb M, Rodgers SS, Lew JI. Appropriate surgical procedure for dominant thyroid nodules of the isthmus $1 \mathrm{~cm}$ or larger. Arch Surg. 2012;147(9):881-884. doi:10.1001/archsurg.2012.728

28. Karatzas T, Charitoudis G, Vasileiadis D, Kapetanakis S, Vasileiadis I. Surgical treatment for dominant malignant nodules of the isthmus of the thyroid gland: a case control study. Int J Surg. 2015;18:64-68. doi:10.1016/j.ijsu.2015.04.039

29. Lee YS, Jeong JJ, Nam K-H, Chung WY, Chang H-S, Park CS. Papillary carcinoma located in the thyroid isthmus. World J Surg. 2010;34(1):36-39. doi:10.1007/s00268-009-0298-6

30. Mai KT, Gulavita P, Lai C, et al. Topographic distribution of papillary thyroid carcinoma by mapping in coronal sections of 125 consecutive thyroidectomy specimens. Int J Surg Pathol. 2014;22(4):303-315. doi:10.1177/1066896913503491

31. Nixon IJ, Palmer FL, Whitcher MM, et al. Thyroid isthmusectomy for well-differentiated thyroid cancer. Ann Surg Oncol. 2011;18 (3):767-770. doi:10.1245/s10434-010-1358-8 
32. Vasileiadis I, Boutzios G, Karalaki M, Misiakos E, Karatzas T. Papillary thyroid carcinoma of the isthmus: total thyroidectomy or isthmusectomy? Am J Surg. 2018;216(1):135-139. doi:10.1016/j. amjsurg.2017.09.008

33. Song CM, Lee DW, Ji YB, Jeong JH, Park JH, Tae K. Frequency and pattern of central lymph node metastasis in papillary carcinoma of the thyroid isthmus. Head Neck. 2016;38(Suppl 1):E412-416. doi:10.1002/hed.24009

34. Hahn SY, Han B-K, Ko EY, Shin JH, Ko ES. Ultrasound findings of papillary thyroid carcinoma originating in the isthmus: comparison with lobe-originating papillary thyroid carcinoma. AJR Am J Roentgenol. 2014;203(3):637-642. doi:10.2214/AJR.13.10746

35. Chai YJ, Kim S-J, Choi JY, Koo DH, Lee KE, Youn Y-K. Papillary thyroid carcinoma located in the isthmus or upper third is associated with Delphian lymph node metastasis. World J Surg. 2014;38 (6):1306-1311. doi:10.1007/s00268-013-2406-X

36. Lee YC, Na SY, Chung H, Kim SI, Eun Y-G. Clinicopathologic characteristics and pattern of central lymph node metastasis in papillary thyroid cancer located in the isthmus. Laryngoscope. 2016;126 (10):2419-2421. doi:10.1002/lary.25926
37. Santrac N, Besic N, Buta M, et al. Lymphatic drainage, regional metastases and surgical management of papillary thyroid carcinoma arising in pyramidal lobe-a single institution experience. Endocr J. 2014;61(1):55-59. doi:10.1507/endocrj.ej13-0316

38. Lee DH, Yoon TM, Kim HK, Lee JK, Kang HC, Lim SC. Intraoperative frozen biopsy of central lymph node in the management of papillary thyroid microcarcinoma. Indian J Otolaryngol Head Neck Surg. 2016;68(1):56-59. doi:10.1007/s12070-0150900-1

39. Raffaelli M, De Crea C, Sessa L, Giustacchini P, Bellantone R, Lombardi CP. Can intraoperative frozen section influence the extension of central neck dissection in $\mathrm{cN0}$ papillary thyroid carcinoma? Langenbecks Arch Surg. 2013;398(3):383-388. doi:10.1007/s00423-012-1036-3

40. Raffaelli M, De Crea C, Sessa L, Fadda G, Bellantone C, Lombardi CP. Ipsilateral central neck dissection plus frozen section examination versus prophylactic bilateral central neck dissection in cN0 papillary thyroid carcinoma. Ann Surg Oncol. 2015;22 (7):2302-2308. doi:10.1245/s10434-015-4383-9

\section{Publish your work in this journal}

Cancer Management and Research is an international, peer-reviewed open access journal focusing on cancer research and the optimal use of preventative and integrated treatment interventions to achieve improved outcomes, enhanced survival and quality of life for the cancer patient.
The manuscript management system is completely online and includes a very quick and fair peer-review system, which is all easy to use. Visit http://www.dovepress.com/testimonials.php to read real quotes from published authors. 